\title{
Ongoing Cortical Activity at Rest: Criticality, Multistability, and Ghost Attractors
}

\author{
Gustavo Deco ${ }^{1,2}$ and Viktor K. Jirsa ${ }^{3}$ \\ ${ }^{1}$ Center for Brain and Cognition, Computational Neuroscience Group, Department of Information and Communication Technologies, Universitat Pompeu \\ Fabra, Barcelona, 08018, Spain, Institució Catalana de la Recerca i Estudis Avançats, Universitat Pompeu Fabra, Barcelona, 08010, Spain, and ${ }^{3}$ Institut de \\ Neurosciences des Systèmes UMR INSERM 1106, Aix-Marseille Université, 13005 Marseille, France
}

The ongoing activity of the brain at rest, i.e., under no stimulation and in absence of any task, is astonishingly highly structured into spatiotemporal patterns. These spatiotemporal patterns, called resting state networks, display low-frequency characteristics $(<0.1 \mathrm{~Hz})$ observed typically in the BOLD-fMRI signal of human subjects. We aim here to understand the origins of resting state activity through modeling via a global spiking attractor network of the brain. This approach offers a realistic mechanistic model at the level of each single brain area based on spiking neurons and realistic AMPA, NMDA, and GABA synapses. Integrating the biologically realistic diffusion tensor imaging/diffusion spectrum imaging-based neuroanatomical connectivity into the brain model, the resultant emerging resting state functional connectivity of the brain network fits quantitatively best the experimentally observed functional connectivity in humans when the brain network operates at the edge of instability. Under these conditions, the slow fluctuating $(<0.1 \mathrm{~Hz})$ resting state networks emerge as structured noise fluctuations around a stable low firing activity equilibrium state in the presence of latent "ghost" multistable attractors. The multistable attractor landscape defines a functionally meaningful dynamic repertoire of the brain network that is inherently present in the neuroanatomical connectivity.

\section{Introduction}

In the absence of external stimulation, any physical system is in its equilibrium state, which is often characterized by the lowest level of activity of that system. Eventually, if noise is inherent in the system, the fluctuations drive the system out of its equilibrium state resulting in low-amplitude random activity. The brain is a particular case of a noisy physical system composed of neurons interconnected through synapses in brain areas, whose activity is mainly characterized by the level of spiking activity in those areas. Nevertheless, the equilibrium state of the brain, i.e., the spontaneous, not stimuli- or task-evoked brain activity during rest, does not reflect just a trivial random activity as one may naively expect. During the last decade, numerous experimental investigations have shown that spontaneous brain activity during rest is highly structured into characteristic spatiotemporal patterns, the socalled resting-state networks (RSNs) (Biswal et al., 1995; Greicius et al., 2003; Fox et al., 2005, 2007; Fransson, 2005; Raichle and Mintun, 2006; Rogers et al., 2007; Vincent et al., 2007).

\footnotetext{
Received May 20, 2011; revised 0ct. 20, 2011; accepted Jan. 2, 2012.

Author contributions: G.D. and V.K.J. designed research; G.D. and V.K.J. performed research; G.D. and V.K.J. contributed unpublished reagents/analytic tools; G.D. and V.K.J. analyzed data; G.D. and V.K.J. wrote the paper.

G.D. was supported by the European Union Grant EC005-024, by SAF2010-16085 and the "La Marato" Foundation, and by the CONSOLIDER-INGENI0 2010 Programme CSD2007-00012. The research reported herein was supported by the Brain Network Recovery Group through the James S. MCDonnell Foundation and the FP7-ICT Brainscales.

The authors declare no competing financial interests.

Correspondence should be addressed to Gustavo Deco, DTIC-Universitat Pompeu Fabra, C/Roc Boronat, 138, 08018 Barcelona, Spain. E-mail: gustavo.deco@upf.edu.

DOI:10.1523/JNEUROSCI.2523-11.2012

Copyright $\odot 2012$ the authors $\quad 0270-6474 / 12 / 323366-10 \$ 15.00 / 0$
}

RSNs reflect the anatomical connectivity between brain areas in a network but cannot be understood in those terms alone (Bullmore and Sporns, 2009). The missing link for understanding the formation and dissolution of RSNs is the dynamics (Deco et al., 2011). Theoretical models allowed us to study the relation between anatomical structure and RSN. In particular, Ghosh et al. (2008) and Deco et al. (2009) were able to explain the formation and dissolution of slowly fluctuating RSNs by considering a simple local oscillatory dynamics at each node. Other RSN modeling efforts included more detailed physiological models for the dynamics of brain areas, but then again imposed specific constraints upon the network dynamics, mostly for reasons of computational efficiency [such as epileptiform node dynamics of a given brain area (Honey et al., 2007) or truncation of time delays via signal transmission and rescaling of the connectivity (Izhikevich and Edelman, 2008)]. A more detailed and complete physiological model for the dynamics of individual brain areas will allow making the link between neurophysiological parameters and RSN dynamics. To this aim, we formulate a detailed global attractor model of the brain network, which offers a realistic mechanistic model at the level of each single brain area based on spiking neurons and realistic AMPA, NMDA, and GABA synapses.

With this in mind, we here demonstrate the emergence of the slowly fluctuating $(<0.1 \mathrm{~Hz}) \mathrm{RSN}$ as noise-driven transient fluctuations around the stable equilibrium state corresponding to low firing activity in all neurons in all areas. The best match between simulated and experimental resting state functional connectivity as measured in humans with fMRI occurs right below the threshold separating the trivial low firing activity equilibrium 
a

Neuroanatomical Connectivity Matrix $b$

\section{Empirical Functional Connectivity Matrix}
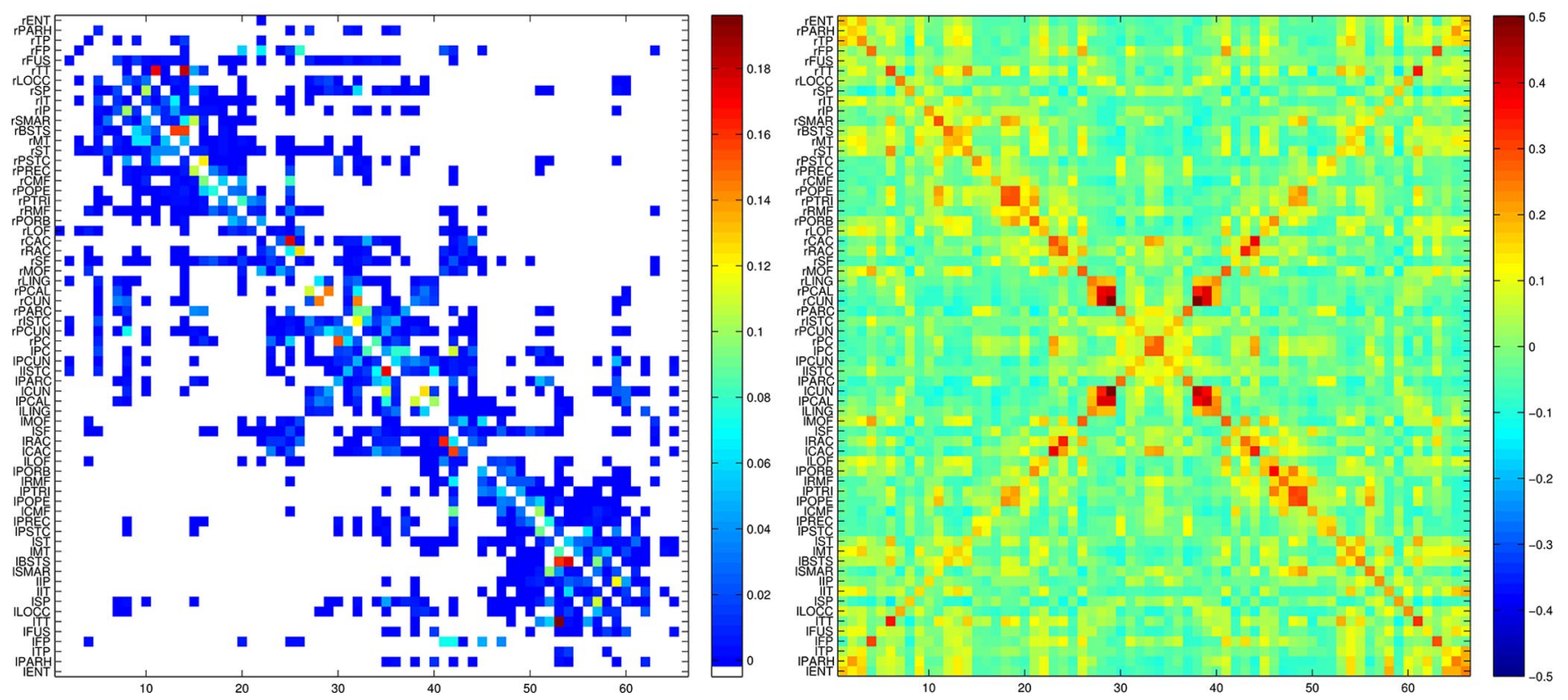

Figure 1. $\quad \boldsymbol{a}$, Neuroanatomical Connectivity matrix obtained by DSI and tractography after averaging across 5 human subjects. $\boldsymbol{b}$, Empirical functional connectivity matrix of the BOLD activity averaged across the same 5 subjects during 20 min under resting state conditions (data from Hagmann et al., 2008).

state from the multistable landscape of attractors with high firing activity in certain brain areas. The latter global attractor structure represents the brain's dynamic repertoire and shows the highest signal complexity as evidenced by entropy.

\section{Materials and Methods}

Empirical neuroanatomical and functional connectivity matrix. Neuroanatomical connections in 5 healthy right-handed male human subjects were extracted by using diffusion spectrum imaging (DSI) white matter tractography (Hagmann et al., 2008; Honey et al., 2009). This neuroanatomical matrix expresses the density with which two different brain areas are connected through white matter fiber tracts, where we used a segmented gray matter parcellation into 66 areas. The neuroanatomical matrix was finally averaged across the 5 human subjects. Figure $1 a$ shows graphically the structure of the connectivity matrix by encoding the strengths of the different connections in a color map. The connectivity matrix is symmetric at the voxel level, due to the fact that tractography cannot distinguish the directionality of the fibers. The internal local connectivity between neurons within a given brain area is considered explicitly in the spiking attractor model adopted (described below), so that at the global level described by the neuroanatomical connectivity the selfconnection of a region to itself is set to 0 in the connectivity matrix. We order the different brain areas in the neuroanatomical connectivity matrix according to modules that have substantially denser connectivity within the module than with the complementary part of the network. Furthermore, homotopic regions in the two cerebral hemispheres were arranged symmetrically with respect to the center of the matrix. This reordering reveals graphically the small-world structure of brain networks through the presentation of clusters of varying size (Watts et al., 1998). In particular, the reordering of the connectivity matrix (Fig. 1a) shows the presence of clusters of nodes that are more connected inside than outside the cluster to which they belong, confirming previous observations (Bullmore and Sporns, 2009).

Resting state activity was also obtained for the same 5 subjects by measuring the corresponding fMRI BOLD signal during $20 \mathrm{~min}$ in absence of stimulation or task. After regressing out the global signal (Fox et al., 2005) and averaging across subjects an empirical func- tional connectivity matrix was obtained (for details, see Honey et al., 2009). This empirical functional connectivity matrix reflects the correlation of the BOLD activity between different brain areas at rest. Figure $1 b$ plots the resulting empirical functional connectivity matrix in a color map.

Global cortical model. The global architecture of the model is shown in Figure 2. Each brain area is modeled by a local spiking attractor network consisting of mutually interconnected populations of excitatory pyramidal neurons and GABAergic inhibitory neurons. This population model serves as the representation of a node in the largescale network, in which the structural connectivity matrix is composed of neuroanatomical connections between distinct brain areas in the human. The structural connectivity is obtained from DSI, which forces the connectivity matrix to be symmetric. This constraint will be only limiting, if the symmetry breaking of the real connectivity is large, since then it may introduce additional oscillatory behaviors of the network (Knock et al., 2009; Jirsa et al., 2010). At the current stage, there is no solution to overcome this constraint, though one could imagine that merging directed structural information from primate data (such as the Cocomac database; Kötter, 2004) with tractographic data from the human might bear promise.

Here, the interconnection between different brain areas is specified by the neuroanatomical human matrix described above. We consider here that the white matter tract connections between two distinct brain areas describe synaptic connections between pyramidal neurons in those areas. We weight those inter-areal connections by the strength specified in the neuroanatomical matrix (numbers of fibers connecting those regions) and by a global factor denoted by $W$, which we will vary systematically to study the dynamics and fix points of the global cortical system (attractors) as a function of it.

Local brain area model: spiking attractor network. For modeling a local brain area (i.e., a node in the global network) we have taken a two-tiered approach, that is modeling the population of single neurons, as well as a mean-field approximation (after Brunel and Wang, 2001).

For the microscopic level of description, we use a biophysically realistic attractor network model consisting of integrate-and-fire spiking neurons with excitatory (AMPA and NMDA) and inhibitory (GABA-A) 
synaptic receptor types (Brunel and Wang, 2001). This type of attractor network of spiking neurons is a dynamical system that in general has the tendency to settle in stationary states, fix points called "attractors," typically characterized by a stable pattern of firing activity. External or even intrinsic noise that appears in the form of finite size effects could provoke destabilization of an attractor inducing therefore transitions between different stable attractors. The dynamics of the network can be described by coupling the dynamical equations describing each neuron and the synaptic variables associated with their mutual coupling. We use here an Integrate-and-Fire (IF) model for describing the spiking activity of neurons, which is characterized by the dynamics of the membrane potential $V(t)$. An IF neuron consists of a basic RC-circuit with the cell membrane capacitance $C_{\mathrm{m}}$ in parallel with a membrane resistance $R_{\mathrm{m}}$. If the membrane potential is below a given threshold $V_{\text {thr }}$ (subthreshold dynamics), then the membrane potential of each neuron in the network is given by the following equation:

$$
C_{\mathrm{m}} \frac{d V(t)}{d t}=-g_{\mathrm{m}}\left(V(t)-V_{\mathrm{L}}\right)-I_{\text {syn }}(t)
$$

where $g_{\mathrm{m}}=1 / R_{\mathrm{m}}$ is the membrane leak conductance, $V_{\mathrm{L}}$ is the resting potential, and $I_{\text {syn }}$ is the synaptic current. The membrane time constant is defined by $\tau_{\mathrm{m}}=C_{\mathrm{m}} / g_{\mathrm{m}}$. When the voltage across the membrane reaches the threshold $V_{\text {thr }}$, the neuron generates a spike, which is then transmitted to other neurons, and then the membrane potential is instantaneously reset to $V_{\text {reset }}$ and maintained there for a refractory time $\tau_{\text {ref, }}$, during which the neuron is unable to produce further spikes.

Incoming input currents coming from connected neurons or from external inputs drive the membrane potential. Indeed, the spikes arriving at a given neural synapse produce an input to the neuron, which induces postsynaptic excitatory or inhibitory potentials essentially given by a low-pass filtering formed through the synaptic receptors. In our case, the total synaptic current is given by the sum of glutamatergic AMPA external excitatory currents $\left(I_{\mathrm{AMPA}, \text { ext }}\right)$, AMPA $\left(I_{\mathrm{AMPA}, \mathrm{rec}}\right)$ and NMDA $\left(I_{\mathrm{NMDA}, \mathrm{rec}}\right)$ recurrent excitatory currents, and GABAergic recurrent inhibitory currents $\left(\mathrm{I}_{\mathrm{GABA}}\right)$ :

$$
I_{\text {syn }}=I_{\mathrm{AMPA}, \mathrm{ext}}+I_{\mathrm{AMPA}, \mathrm{rec}}+I_{\mathrm{NMDA}, \mathrm{rec}}+I_{\mathrm{GABA}},
$$

where

$$
I_{\mathrm{AMPA}, \mathrm{ext}}(t)=g_{\mathrm{AMPA}, \mathrm{ext}}\left(V(t)-V_{\mathrm{E}}\right) \sum_{j=1}^{\mathrm{N}_{\text {ext }}} s_{j}^{\mathrm{AMPA}, \mathrm{ext}}(t),
$$

$I_{\mathrm{AMPA}, \mathrm{rec}}(t)=g_{\mathrm{AMPA}, \mathrm{rec}}\left(V(t)-V_{\mathrm{E}}\right)$

$$
\times \sum_{j=1}^{\mathrm{N}_{\mathrm{E}}} w_{j} s_{j}^{\mathrm{AMPA}, \mathrm{rec}}(t)
$$

$I_{\mathrm{NMDA}, \mathrm{rec}}(t)=\frac{g_{\mathrm{NMDA}, \mathrm{rec}}\left(V(t)-V_{\mathrm{E}}\right)}{1+\gamma e^{-\beta V(t)}}$

$$
\times \sum_{j=1}^{\mathrm{N}_{\mathrm{E}}} w_{j} \mathrm{~s}_{j}^{\mathrm{NMDA}, \mathrm{rec}}(t)
$$

$I_{\mathrm{GABA}}(t)=g_{\mathrm{GABA}}\left(V(t)-V_{\mathrm{I}}\right)$
Here $g_{\mathrm{AMPA}, \mathrm{ext}}, g_{\mathrm{AMPA}, \mathrm{rec}}, g_{\mathrm{NMDA}, \mathrm{rec}}$, and $g_{\mathrm{GABA}}$ are the synaptic conductances, and $V_{\mathrm{E}}, V_{\mathrm{I}}$ the excitatory and inhibitory reversal potentials, respectively. The dimensionless parameters $w_{\mathrm{j}}$ of the connections are the synaptic weights. The NMDA currents are voltage dependent and they are modulated by intracellular magnesium concentrations. The gating variables $s_{j}^{i}(t)$ are the fractions of open channels of neurons and are given by:

$$
\begin{gathered}
\frac{d s_{j}^{\mathrm{AMPA}, \mathrm{ext}}(t)}{d t}=-\frac{s_{j}^{\mathrm{AMPA}, \mathrm{ext}}(t)}{\tau_{\mathrm{AMPA}}}+\sum_{k} \delta\left(t-t_{j}^{k}\right), \\
\frac{d s_{j}^{\mathrm{AMPA}, \mathrm{rec}}(t)}{d t}=-\frac{s_{j}^{\mathrm{AMPA}, \mathrm{rec}}(t)}{\tau_{\mathrm{AMPA}}}+\sum_{k} \delta\left(\mathrm{t}-\mathrm{t}_{j}^{k}\right), \\
\frac{d s_{j}^{\mathrm{NMDA}, \mathrm{rec}}(t)}{d t}=-\frac{s_{j}^{\mathrm{NMDA}, \mathrm{rec}}(t)}{\tau_{\mathrm{NMDA}, \mathrm{decay}}}+\alpha x_{j}(t)\left(1-s_{j}^{\mathrm{NMDA}, \mathrm{rec}}(t)\right), \\
\frac{d x_{j}^{\mathrm{NMDA}, \mathrm{rec}}(t)}{d t}=-\frac{x_{j}^{\mathrm{NMDA}, \mathrm{rec}}(t)}{\tau_{\mathrm{NMDA}, \mathrm{rise}}}+\sum_{k} \delta\left(t-t_{j}^{k}\right), \\
\frac{d s_{j}^{\mathrm{GABA}}(t)}{d t}=-\frac{s_{j}^{\mathrm{GABA}}(t)}{\tau_{\mathrm{GABA}}}+\sum_{k} \delta\left(t-t_{j}^{k}\right) .
\end{gathered}
$$

The sums over the index $k$ represent all the spikes emitted by the presynaptic neuron $j$ (at times $t_{j}^{k}$ ). In Equations $7-11, \tau_{\mathrm{NMDA}, \text { rise }}$ and $\tau_{\mathrm{NMDA} \text {,decay }}$ are the rise and decay times for the NMDA synapses, and $\tau_{\mathrm{AMPA}}$ and $\tau_{\mathrm{GABA}}$ the decay times for AMPA and GABA synapses. The rise times of both AMPA and GABA synaptic currents are neglected because they are short $(<1 \mathrm{~ms})$. The values of the constant parameters and default values of the free parameters used in the simulations are displayed in Table 1.

Each local attractor network contains 100 excitatory pyramidal neurons and 100 inhibitory neurons. The total cortical network has, therefore, $66 \times 200=13,200$ neurons. We use local attractor networks where neurons are organized into two sets of populations (Fig. 2), namely: an inhibitory population, and an excitatory population. The network is fully connected, meaning that each neuron in the network receives $N_{\mathrm{E}}$ excit- 
Table 1. Neural and synaptic parameters for each local brain area

\begin{tabular}{|c|c|c|c|c|c|}
\hline \multicolumn{2}{|c|}{ Excitatory neurons } & \multicolumn{2}{|c|}{ Inhibitory neurons } & \multicolumn{2}{|l|}{ Synapses } \\
\hline$N_{\mathrm{E}}$ & 100 neurons & $N_{1}$ & 100 neurons & $V_{\mathrm{E}}$ & $0 \mathrm{mV}$ \\
\hline$C_{m}$ & $0.5 \mathrm{nF}$ & $C_{m}$ & $0.2 \mathrm{nF}$ & $V_{1}$ & $-70 \mathrm{mV}$ \\
\hline$g_{\mathrm{m}}$ & $25 \mathrm{nS}$ & $g_{\mathrm{m}}$ & $20 \mathrm{nS}$ & $\tau_{\text {AMPA }}$ & $2 \mathrm{~ms}$ \\
\hline$V_{\mathrm{L}}$ & $-70 \mathrm{mV}$ & $V_{\mathrm{L}}$ & $-70 \mathrm{mV}$ & $\tau_{\mathrm{NMDA} \text {,rise }}$ & $2 \mathrm{~ms}$ \\
\hline$V_{\text {thr }}$ & $-50 m V$ & $V_{\text {thr }}$ & $-50 \mathrm{mV}$ & $\tau_{\text {NMDA,decay }}$ & $100 \mathrm{~ms}$ \\
\hline$V_{\text {reset }}$ & $-55 \mathrm{mV}$ & $V_{\text {reset }}$ & $-55 \mathrm{mV}$ & $\tau_{\mathrm{GABA}}$ & $10 \mathrm{~ms}$ \\
\hline$\tau_{\text {ref }}$ & $2 \mathrm{~ms}$ & $\tau_{\text {ref }}$ & $1 \mathrm{~ms}$ & $\alpha$ & $0.5 \mathrm{kHz}$ \\
\hline$g_{\text {AMPA,ext }}$ & $2.496 \mathrm{nS}$ & $g_{\text {AMPA, ext }}$ & $1.944 \mathrm{nS}$ & $\beta$ & 0.062 \\
\hline$g_{\text {AMPA,rec }}$ & $0.104 \mathrm{nS}$ & $g_{\text {AMPA,rec }}$ & $0.081 \mathrm{nS}$ & $\gamma$ & 0.28 \\
\hline$g_{\mathrm{NMDA}, \mathrm{rec}}$ & $0.327 \mathrm{nS}$ & $g_{\mathrm{NMDA}, \mathrm{rec}}$ & $0.258 \mathrm{nS}$ & & \\
\hline$g_{\text {GABA }}$ & $4.375 \mathrm{nS}$ & $g_{\text {GABA }}$ & $3.4055 \mathrm{nS}$ & & \\
\hline
\end{tabular}

atory and $N_{\mathrm{I}}$ inhibitory synaptic contacts. The connection strengths between and within the populations are determined by dimensionless weights $w_{\mathrm{j}}$. The recurrent self-excitation within each excitatory population is given by the weight $w_{+}$, and within each inhibitory population is given by the weight $w=1$. The connections between excitatory and inhibitory neurons have the weight $w=1$.

All neurons in the network always receive an external background input from $N_{\text {ext }}=800$ external neurons emitting uncorrelated Poisson spike trains. More specifically, and for all neurons inside a given population $p$, the resulting global spike train, which is still Poissonian, has a time-varying rate $\nu_{\text {ext }}^{p}(t)$, governed by

$$
\tau_{\mathrm{n}} \frac{d \nu_{\mathrm{ext}}^{p}(t)}{d t}=-\left(\nu_{\mathrm{ext}}^{p}(t)-\nu_{0}\right)+\sigma_{\mathrm{v}} \sqrt{2 \tau_{n}} n^{p}(t),
$$

where $t_{\mathrm{n}}=300 \mathrm{~ms}, \nu_{0}=2.4 \mathrm{kHz}, \sigma_{\nu}$ is the SD of $\nu_{\text {ext }}^{p}(t)$, and $n^{\mathrm{P}}(t)$ is normalized Gaussian white noise. Negative values of $\nu_{\text {ext }}^{p}(t)$ that could arise due to the noise term are rectified to zero. These input rate fluctuations represent the noisy fluctuations that are typically observed in vivo.

Mean-field reduction. For the mesoscopic level of description, the mean-field approximation reduces the number of integration variables to one for each neural population (Brunel and Wang, 2001). Solving the mean-field equations provides the fixed points of the population firing rates, i.e., the stationary states of the populations after the period of dynamical transients. As this can be done much more quickly than integrating the full spiking model, scanning the parameter space to find a parameter set matching the experimental findings becomes feasible. Other mean-field approaches consider parameter dispersion (Assisi et al., 2005; Stefanescu and Jirsa, 2008) and different types of coupling including gap junctions and chemical synapses (Jirsa and Stefanescu, 2011; Stefanescu and Jirsa, 2011), which typically results in a higherdimensional state vector and hence is computationally more expensive.

In the mean-field formulation the potential of a neuron is calculated according to:

$$
\tau_{x} \frac{d V(t)}{d t}=-V(t)+\mu_{x}+\sigma_{x} \sqrt{\tau_{x}} \eta(t)
$$

where $V(t)$ is the membrane potential, $x$ labels the populations, $\tau_{x}$ is the effective membrane time constant, $\mu_{x}$ is the mean value of the membrane potential in absence of spiking and fluctuations, $\sigma_{x}$ measures the magnitude of the fluctuations and $\eta$ is a Gaussian process with absolute exponentially decaying correlation function and time constant $\tau_{\mathrm{AMPA}}$. The quantities $\mu_{x}$ and $\sigma_{x}$ are given by:

$$
\begin{gathered}
\mu_{x}=\frac{\begin{array}{c}
\left(T_{\text {ext }} \nu_{\text {ext }}+T_{\text {AMPA }} n_{x}^{\mathrm{AMPA}}+\rho_{1} n_{x}^{\mathrm{NMDA}}\right) V_{\mathrm{E}}+ \\
\rho_{2} n_{x}^{\mathrm{NMDA}}\langle V\rangle+T_{\mathrm{I}} n_{x}^{\mathrm{GABA}} V_{\mathrm{I}}+V_{\mathrm{L}}
\end{array}}{S_{x}}, \\
\sigma_{x}^{2}=\frac{g_{\mathrm{AMPA}, \mathrm{ext}}^{2}\left(\langle V\rangle-V_{\mathrm{E}}\right)^{2} N_{\mathrm{ext}} \nu_{\mathrm{ext}} \tau_{\mathrm{AMPA}}^{2} \tau_{x}}{g_{\mathrm{m}}^{2} \tau_{\mathrm{m}}^{2}},
\end{gathered}
$$

where $\nu_{\text {ext }}$ is the external incoming spiking rate, $\nu_{\mathrm{I}}$ is the spiking rate of the inhibitory population, $\tau_{\mathrm{m}}=C_{\mathrm{m}} / g_{\mathrm{m}}$ with the values for the excitatory or inhibitory neurons depending of the population considered. The other quantities are given by:

$$
\begin{aligned}
& S_{x}=1+T_{\mathrm{ext}} \nu_{\mathrm{ext}}+T_{\mathrm{AMPA}} n_{x}^{\mathrm{AMPA}}+\left(\rho_{1}+\rho_{2}\right) n_{x}^{\mathrm{NMDA}}+T_{\mathrm{I}} n_{x}^{\mathrm{GABA}}, \\
& \tau_{x}=\frac{C_{\mathrm{m}}}{g_{\mathrm{m}} S_{x}} \\
& n_{x}^{\mathrm{AMPA}}=\sum_{j=1}^{\mathrm{p}} f_{j} w_{j x}^{\mathrm{AMPA}} \nu_{j} \\
& n_{x}^{\mathrm{NMDA}}=\sum_{j=1}^{p} f_{j} w_{j x}^{\mathrm{NMDA}} \psi\left(\nu_{j}\right) \\
& n_{x}^{\mathrm{GABA}}=\sum_{j=1}^{\mathrm{p}} f_{j} w_{j x}^{\mathrm{GABA}} \nu_{j} \\
& \psi(\nu)=\frac{\nu \tau_{\mathrm{NMDA}}}{1+\nu \tau_{\mathrm{NMDA}}}\left(1+\frac{1}{1+\nu \tau_{\mathrm{NMDA}}} \sum_{n=1}^{\infty} \frac{\left(-\alpha \tau_{\mathrm{NMDA}, \mathrm{rise}}\right)^{n} T_{n}(\nu)}{(n+1) !}\right) \\
& T_{n}(\nu)=\sum_{k=0}^{n}(-1)^{k}\left(\begin{array}{l}
n \\
k
\end{array}\right) \frac{\tau_{\mathrm{NMDA}, \text { rise }}\left(1+\nu \tau_{\mathrm{NMDA}}\right)}{\tau_{\mathrm{NMDA}, \mathrm{rise}}\left(1+\tau_{\mathrm{NMDA}}\right)+k \tau_{\mathrm{NMDA}, \mathrm{decay}}}, \\
& \tau_{\mathrm{NMDA}}=\alpha \tau_{\mathrm{NMDA}, \mathrm{rise}} \tau_{\mathrm{NMDA}, \mathrm{decay}} \\
& T_{\mathrm{ext}}=\frac{g_{\mathrm{AMPA}, \mathrm{ext}} \tau_{\mathrm{AMPA}}}{g_{\mathrm{m}}}, \\
& T_{\mathrm{AMPA}}=\frac{g_{\mathrm{AMPA}, \mathrm{rec}} N_{\mathrm{E}} \tau_{\mathrm{AMPA}}}{g_{\mathrm{m}}}, \\
& \rho_{1}=\frac{g_{\mathrm{NMDA}} N_{\mathrm{E}}}{g_{\mathrm{m}} J}, \\
& \rho_{2}=\beta \frac{g_{\mathrm{NMDA}} N_{\mathrm{E}}\left(\left\langle V_{x}\right\rangle-V_{\mathrm{E}}\right)(J-1)}{g_{\mathrm{m}} J^{2}}, \\
& J=1+\gamma \exp \left(-\beta\left\langle V_{x}\right\rangle\right) \\
& T_{\mathrm{I}}=\frac{g_{\mathrm{GABA}} N_{\mathrm{I}} \tau_{\mathrm{GABA}}}{g_{\mathrm{m}}}, \\
& \left\langle V_{x}\right\rangle=\mu_{x}-\left(V_{\mathrm{thr}}-V_{\text {reset }}\right) \nu_{x} \tau_{x},
\end{aligned}
$$

where $p$ is the number of excitatory populations, $f_{x}$ is the fraction of neurons in the excitatory population $x, \omega_{j, x}$ the weight of the connections from population $x$ to population $j, \nu_{x}$ is the spiking rate of the excitatory population $x, \gamma=0.28, \beta=0.062$, and the average membrane potential $\left\langle\mathrm{V}_{x}\right\rangle$ has a value between $-55 \mathrm{mV}$ and $-50 \mathrm{mV}$.

The mean-field approximation finally yields a set of $n$ nonlinear equations describing the average firing rates of the different populations in the network as a function of the defined quantities $\mu_{\mathrm{x}}$ and $\sigma_{\mathrm{x}}$ :

$$
\nu_{x}=\phi\left(\mu_{x}, \sigma_{x}\right), \quad x=1, \ldots, n,
$$

where $\phi$ is the transduction function of population $x$, which gives the output rate of a population $x$ in terms of the inputs, which in turn depend on the rates of all the populations. 


$$
\begin{gathered}
\phi\left(\mu_{x}, \sigma_{x}\right)=\left(\tau_{\mathrm{rp}}+\tau_{x} \int_{\beta\left(\mu_{x}, \sigma_{x}\right)}^{\alpha\left(\mu_{x}, \sigma_{x}\right)} d u \sqrt{\pi} \exp \left(u^{2}\right)[1+\operatorname{erf}(u)]\right)^{-1}, \\
\alpha\left(\mu_{x}, \sigma_{x}\right)=\frac{\left(V_{\mathrm{thr}}-\mu_{x}\right)}{\sigma_{x}}\left(1+0.5 \frac{\tau_{\mathrm{AMPA}}}{\tau_{x}}\right)+1.03 \sqrt{\frac{\tau_{\mathrm{AMPA}}}{\tau_{x}}}-0.5 \frac{\tau_{\mathrm{AMPA}}}{\tau_{x}}, \\
\beta\left(\mu_{x}, \sigma_{x}\right)=\frac{\left(V_{\text {reset }}-\mu_{x}\right)}{\sigma_{x}},
\end{gathered}
$$

with $\operatorname{erf}(u)$ the error function and $\tau_{r p}$ the refractory period which is considered to be $2 \mathrm{~ms}$ for excitatory neurons and $1 \mathrm{~ms}$ for inhibitory neurons. To solve the equations defined by Equation 31 for all $x$, we numerically integrate Equations 32-34 and the differential equation below, whose fixed-point solutions correspond to solutions to Equation 31 :

$$
\tau_{x} \frac{d \nu_{x}}{d t}=-\nu_{x}+\phi\left(\mu_{x}, \sigma_{x}\right)
$$

To find the possible fixed points that coexist for a given parameter set, Equation 35 has to be integrated for different initial conditions of population firing rates over a range of external inputs. Generally, the firing rates obtained by the mean-field approximation would be exact if the number of neurons was infinitely large and the unitary postsynaptic potentials elicited by presynaptic spikes were infinitesimally small.

With the conductivity values specified in Table 1 , the values of $w_{+}$ studied ranged between 1.1 and 1.7, such that the local brain area network, when isolated from the rest of the cortex, has a unique stable attractor, namely the spontaneous state attractor, where all excitatory neurons spike asynchronously at a rate of $3 \mathrm{~Hz}$ and the inhibitory neurons at a rate of $9 \mathrm{~Hz}$ (Brunel and Wang, 2001). We also studied the effect of different levels of noise from $\sigma_{\nu}=0.1-0.4$. The results are robust and not dependent of the values of $w_{+}$and $\sigma_{\nu}$. In the Results section we show the simulations by using always $w_{+}=1.5$ and $\sigma_{\nu}=0.14$.

BOLD-fMRI signal. The simulation of the fMRI BOLD signal in the global cortical model is computed by means of the Balloon-Windkessel hemodynamic model of (Friston et al., 2003). The Ballon-Windkessel model describes the coupling of perfusion to BOLD signal, with a dynamical model of the transduction of neural activity into perfusion changes. The model assumes that the BOLD signal is a static nonlinear function of the normalized total deoxyhemoglobin voxel content, normalized venous volume, resting net oxygen extraction fraction by the capillary bed, and resting blood volume fraction. The BOLD-signal estimation for each brain area is computed by the level of neuronal activity summed over all neurons in both populations (excitatory and inhibitory populations) in that particular area. In all our simulations shown here this level of neuronal activity is given by the rate of spiking activity in windows of $1 \mathrm{~ms}$. In brief, for the $i$ th region, neuronal activity $z_{i}$ causes an increase in a vasodilatory signal $s_{i}$ that is subject to autoregulatory feedback. Inflow $f_{i}$ responds in proportion to this signal with concomitant changes in blood volume $v_{i}$ and deoxyhemoglobin content $q_{i}$. The equations relating these biophysical variables are:

$$
\begin{gathered}
\frac{d s_{i}(t)}{d t}=z_{i}-k_{i} s_{i}-\gamma_{i}\left(f_{i}-1\right), \\
\frac{d f_{i}(t)}{d t}=s_{i}, \\
\tau_{i} \frac{d \nu_{i}(t)}{d t}=f_{i}-\nu_{i}^{1 / \alpha}, \\
\tau_{i} \frac{d q_{i}(t)}{d t}=\frac{f_{i}\left(1-\left(1-\rho_{i}\right)^{1 / f_{i}}\right)}{\rho_{i}}-\frac{q_{i} \nu_{i}^{1 / \alpha}}{\nu_{i}},
\end{gathered}
$$

where $\rho$ is the resting oxygen extraction fraction. The BOLD signal is taken to be a static nonlinear function of volume and deoxyhemoglobin that comprises a volume-weighted sum of extra- and intravascular signals:

$$
y_{i}=V_{0}\left(7 \rho_{i}\left(1-q_{i}\right)+2\left(1-\frac{q_{i}}{\nu_{i}}\right)+\left(2 \rho_{i}-0.2\right)\left(1-\nu_{i}\right)\right),
$$

where $V_{0}=0.02$ is the resting blood volume fraction. The biophysical parameters were taken as in (Friston et al., 2003). We also tested simulations in which the activity $z_{i}$ is given by the glutamate turnover (Logothetis et al., 2001; Attwell et al., 2010) and obtained equivalent results, since the synaptic activity typically strongly correlates with the spiking activity in our network.

Entropy. To explore how the dynamics depend on $W$ (scaling of the global inter-areal coupling strength) the mean-field equations are solved for particular values of $W$ starting from different initial conditions. These initial conditions are chosen at random for each node by initially setting the average firing rate of the selective excitatory pool randomly to $10 \mathrm{~Hz}$ (active) or $3 \mathrm{~Hz}$ (inactive) and the average firing rate of the inhibitory pool to $9 \mathrm{~Hz}$. To determine at which value for $W$ a bifurcation has occurred the number of attractors and the entropy of the system is derived from the final average firing rates of the selective pools after 4000 steps found for each initial condition. If the final average firing rate of each selective pool is self-similar across initial conditions; i.e., the final average firing rate of each selective pool after initial condition $X$ does not differ from any of the final average firing rates of this same pool by $>10 \mathrm{~Hz}$, there is only a single attractor. If this is not the case, the number of attractors can be established by counting the number of initial conditions in which at least one selective pool has a different final average firing rate from all previously found final average firing rates of that same pool. In the case of only a single attractor the entropy is 0 as the system will invariably settle within it. If, however, the number of attractors is larger than 1 , the entropy is given by

$$
H=-\sum_{i} p(i) \log p(i)
$$

where $p(i)$ is the probability that the system settles in attractor $i$.

\section{Results}

We consider the dynamics of a realistic global attractor spiking network structured in brain areas, which are interconnected according the diffusion tensor imaging (DTI)/DSI-based neuroanatomical connectivity matrix of the brain of five human subjects (Human Connectome; Hagmann et al., 2008; see Materials and Methods). Precisely, the interplay between the particular underlying structure of the neuroanatomical inter-areal connections, the local attractor spiking dynamics of each area and the noise is able to explain the generation of the spatiotemporal structured resting state networks evidenced by the fMRI functional connectivity gained from the same subjects. In the following we will demonstrate that resting state networks in fMRI result from structured noise fluctuations around the trivial low firing equilibrium state induced at the edge of a bifurcation by the presence of latent "ghost" multistable attractors corresponding to distinct foci of high firing activity in particular brain areas.

We first study the stationary fix points (attractor landscape) of the cortical spiking network described in the Materials and Methods. We reduce the spiking dynamics to a set of mean-field equations that describes the stationary states (see Materials and Methods). In particular, we study the attractors of the global cortical system as a function of the parameter $W$ scaling the global inter-areal coupling strength. Figure 3 plots the number of attractors found in the system as a function of $W$. We find these attrac- 


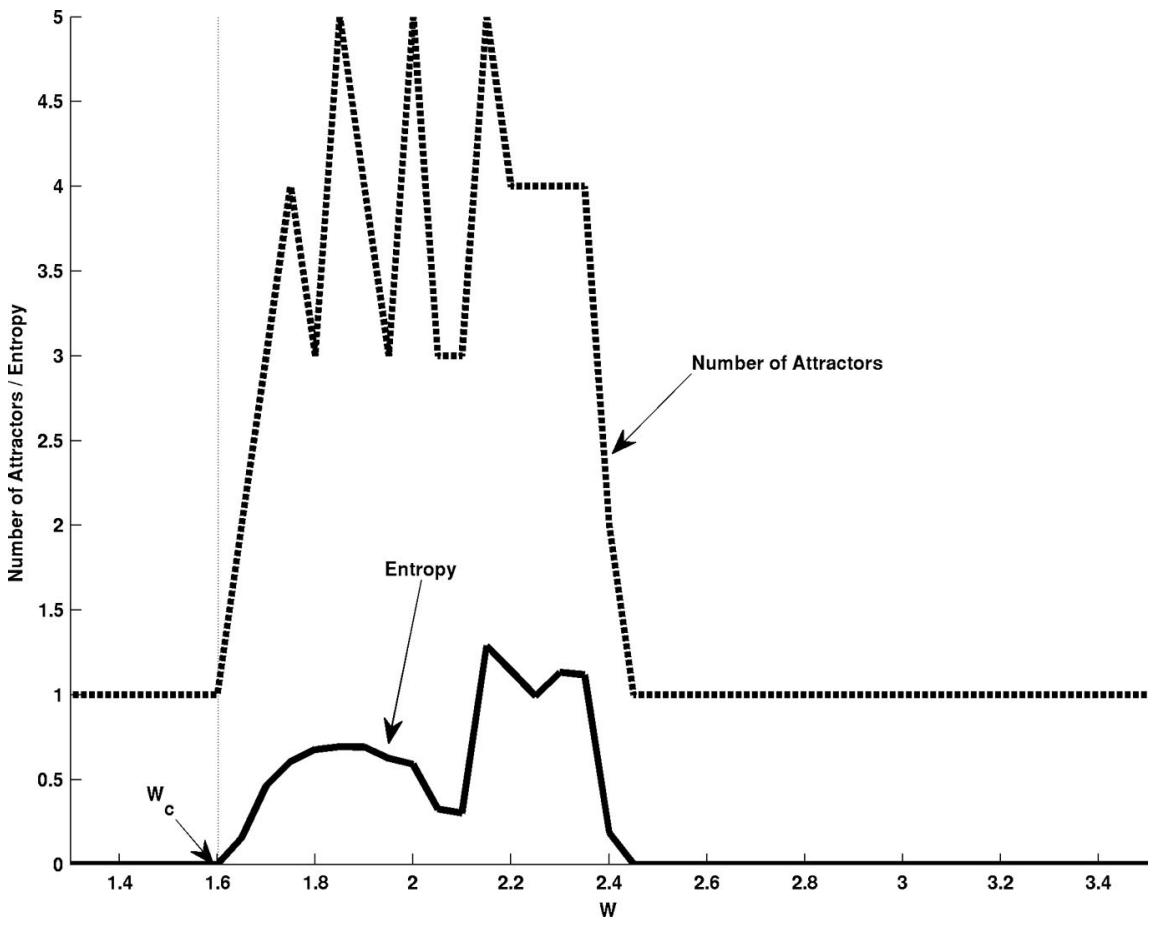

Figure 3. Mean-field analyses of the attractor landscape of the cortical spiking network as a function of the global inter-areal coupling strength. The dashed line plots the number of stable attractors, whereas the continued line shows the entropy of the attractors.

a

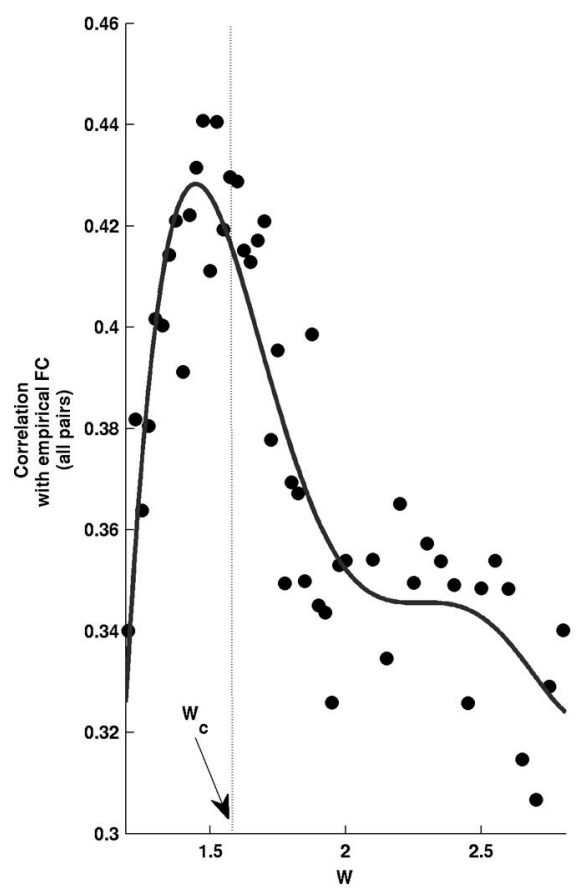

b

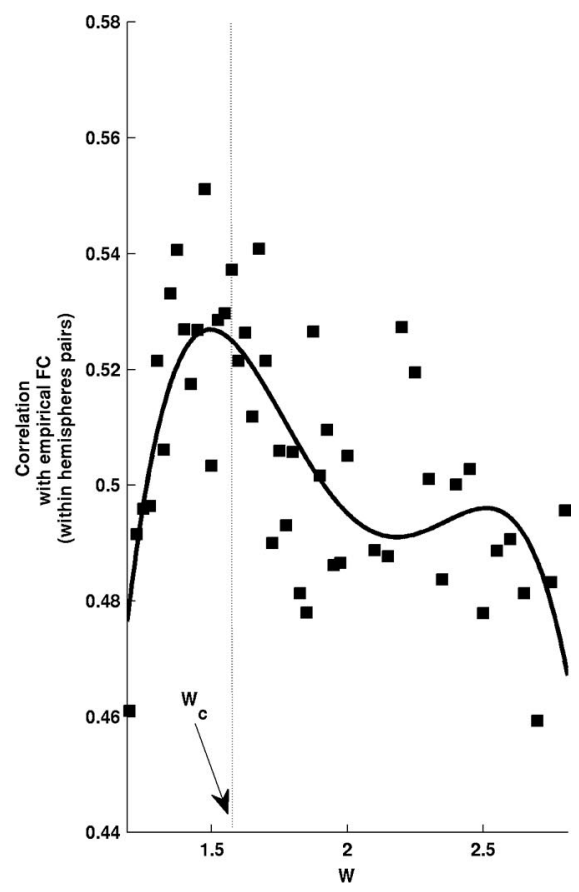

Figure 4. Fitting of empirical data as measured by the correlation between simulated and empirical functional connectivity as a function of the global coupling parameter $W$. The best fit is achieved at the edge of the bifurcation.

tors by iterating as usual the mean-field reduced rate equations initialized with 1000 different initial conditions. Furthermore, Figure 3 also shows the entropy of the attractors calculated by considering the probability of obtaining the different attractors after departing from random initial conditions (see Materials and Methods).

This entropy characterizes the effective expected variability of cortical activity due to nonstationary noise-driven transitions between multistable attractors (which will be studied later with the full spiking implementation). For very small values of $W$ only one attractor is stable and therefore the entropy is zero. That attractor corresponds to the trivial spontaneous ground state of the system where all neurons in all brain areas are firing at a low level of activity (excitatory neuron at $3 \mathrm{~Hz}$ and inhibitory neurons at $9 \mathrm{~Hz}$ ). For very large values of $W$ also only one attractor is stable and therefore the entropy again turns to zero. That attractor corresponds to the "epileptiform" case where all excitatory neurons are highly activated in all brain areas. In intermediate regions of $W$ multistability of many attractors corresponding to distinct foci of high firing activity in particular brain areas emerges, causing consequently an increase of the entropy. For our further analysis, the bifurcation separating the trivial spontaneous state and the multistability region at $W=W_{c}=$ 1.6 will be particularly relevant.

To identify the mechanisms underlying resting state generation, we study next how the dynamics of the model depends on the global coupling strength $W$. The dynamics of the cortical model is simulated by using the spiking model. We first calculate the firing activity in all brain areas, and then simulate the BOLD-fMRI signal by using the Balloon-Windkessel model (Friston et al., 2003; see Materials and Methods). Then the simulated BOLD signal was downsampled at $2 \mathrm{~s}$ to have the same resolution as in (Honey et al., 2009) and the global signal (average over all regions) (Fox et al., 2005, 2007) was regressed out of the BOLD time series. Finally, we computed the simulated functional connectivity by calculating the correlation matrix of the BOLD activity between all brain areas. To identify the region of the parameter $W$ where the model best reproduces the empirical functional connectivity, we computed the Pearson correlation between both the empirical and the simulated functional connectivity matrix. Figure 4 shows how the fitting of empirical data as measured by the correlation between simulated and empirical functional connectivity is maximal for values of $W$ at the edge of the bifurcation found in Figure 3. Interhemispherical correlations are much weaker and sometimes missed in the model, because the DTI/DSI tractography also missed those connections in the neuroanatomical matrix that we used (see Fig. 1a). Figure $4 a$ plots the fitting 


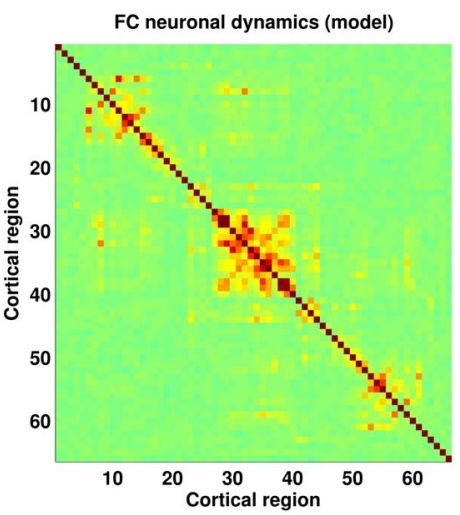

a

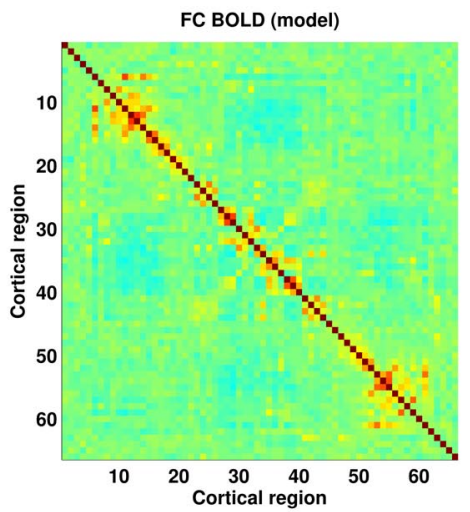

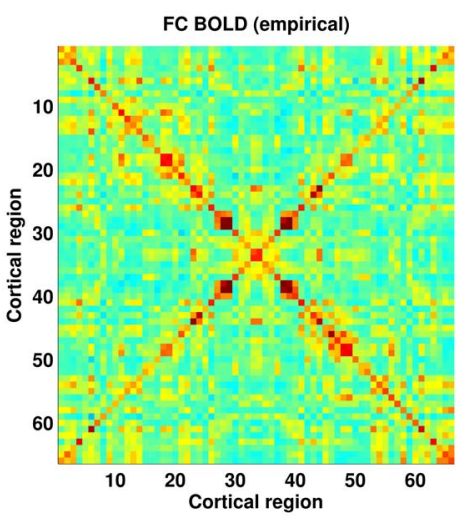

Seed $=$ IPC

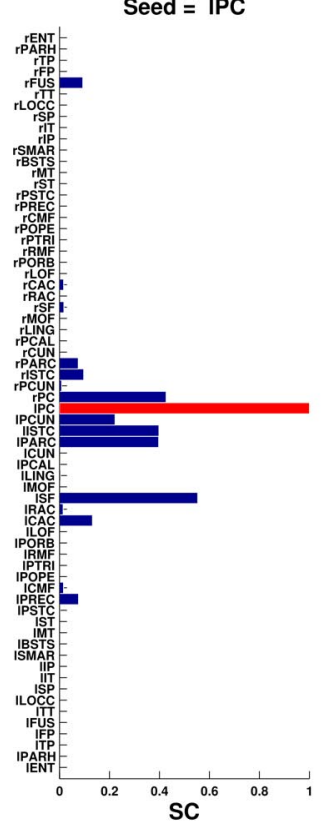

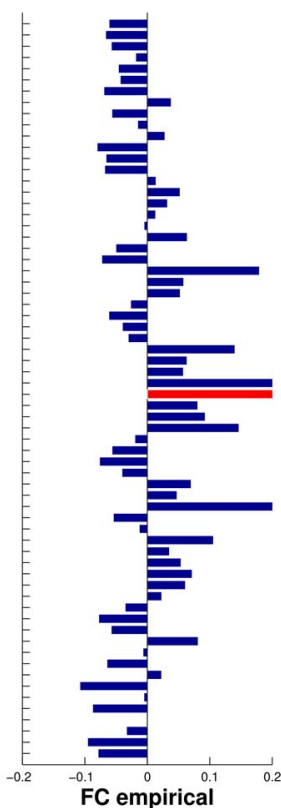

b

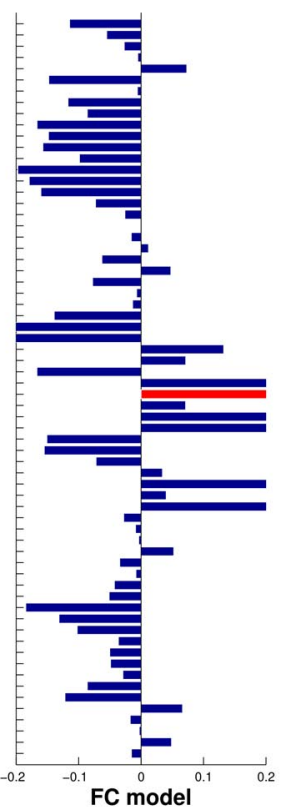

FC model

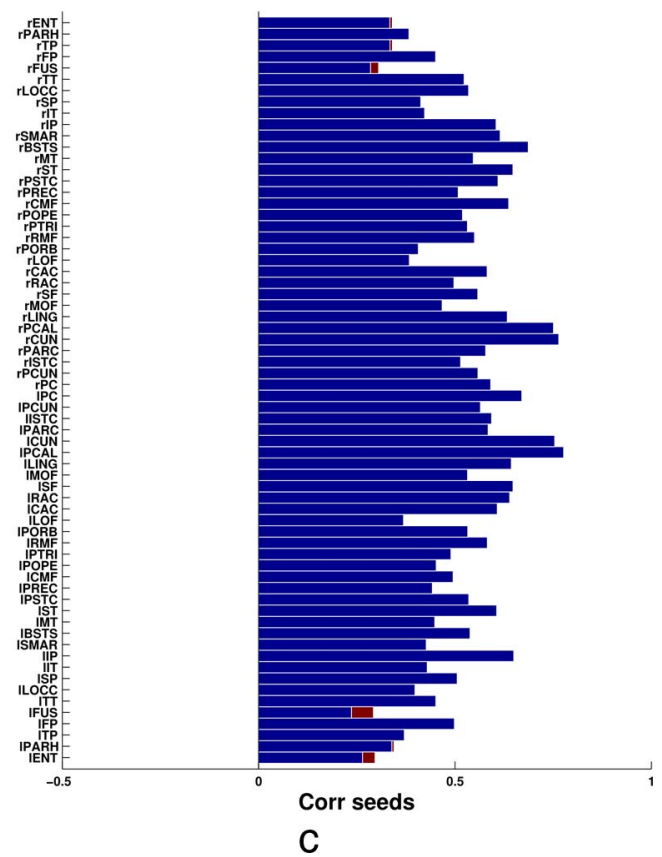

Figure 5. Detailed comparison between the neuroanatomical connectivity matrix, the empirical and the simulated functional connectivity for the working point $W=W_{c}=1.6$ at the edge of the bifurcation. $\boldsymbol{a}$, Left, Functional connectivity matrix based on the firing rates dynamics. Middle, Functional connectivity matrix based on the simulated BOLD signal. Right, Empirical functional connectivity. $\boldsymbol{b}$, From left to right: neuroanatomical connections, empirical and simulated functional connectivity for the seed IPC. $\boldsymbol{c}$, Pearson correlation between empirical and simulated functional connectivity for each individual seed (blue), and the corresponding $p$-value (red).

of the functional connectivity at rest between theory and empirical data taking into account all pairs, i.e., including also the interhemispherical, whereas Figure $4 b$ plots the results for single hemispheres, i.e., excluding interhemispherical pairs.

For the particular value $W=W_{c}$, Figure 5 shows a detailed comparison between the simulated and empirical functional connectivity. Figure $5 a$ shows the simulated (left and middle subpanel) and empirical (right subpanel) functional connectivity matrices. In particular, for the simulated model we present the functional connectivity matrix based on the firing rate dynamics (left subpanel) and on the simulated BOLD signal (middle subpanel). The highest empirical functional connectivity correlations correspond mainly to pairs linked by the neuroanatomical connectivity matrix (Honey et al., 2009). The best agreement occurs when the simulated correlation is also high for these pairs. Figure $5 b$ presents the neuroanatomical connectivity matrix (left subpanel), the empirical (middle subpanel) and the simulated (right subpanel) functional connectivity between one seed region and all other brain regions. We took the left posterior cingulated (IPC) as a seed (red bars in the plots). The simulated functional connectivity largely reproduces a lot of details of the empirical functional connectivity between the Default Mode Network regions (Greicius et al., 2003) along the medial axis. Figure $5 c$ shows that Pearson correlation between both functional connectivity matrices for a given seed is significantly positive for all seeds.

For the same working point, at the edge of the bifurcation, Figure 6 shows typical temporal evolution of the simulated BOLD signal for three particular brain regions. We took two regions (IPC and left superior frontal (ISF)) in the Default Mode Network which are correlated and a third region in temporal (IST) brain areas (Attentional Network) which is anticorrelated with the first two regions. The model is able to capture the correlation of BOLD activity between brain regions in the same network and the anticorrelation between brain region in different resting state networks as evidenced previously by Fox et al. (2005). Further more, these patterns of spatiotemporal correlations defining the 


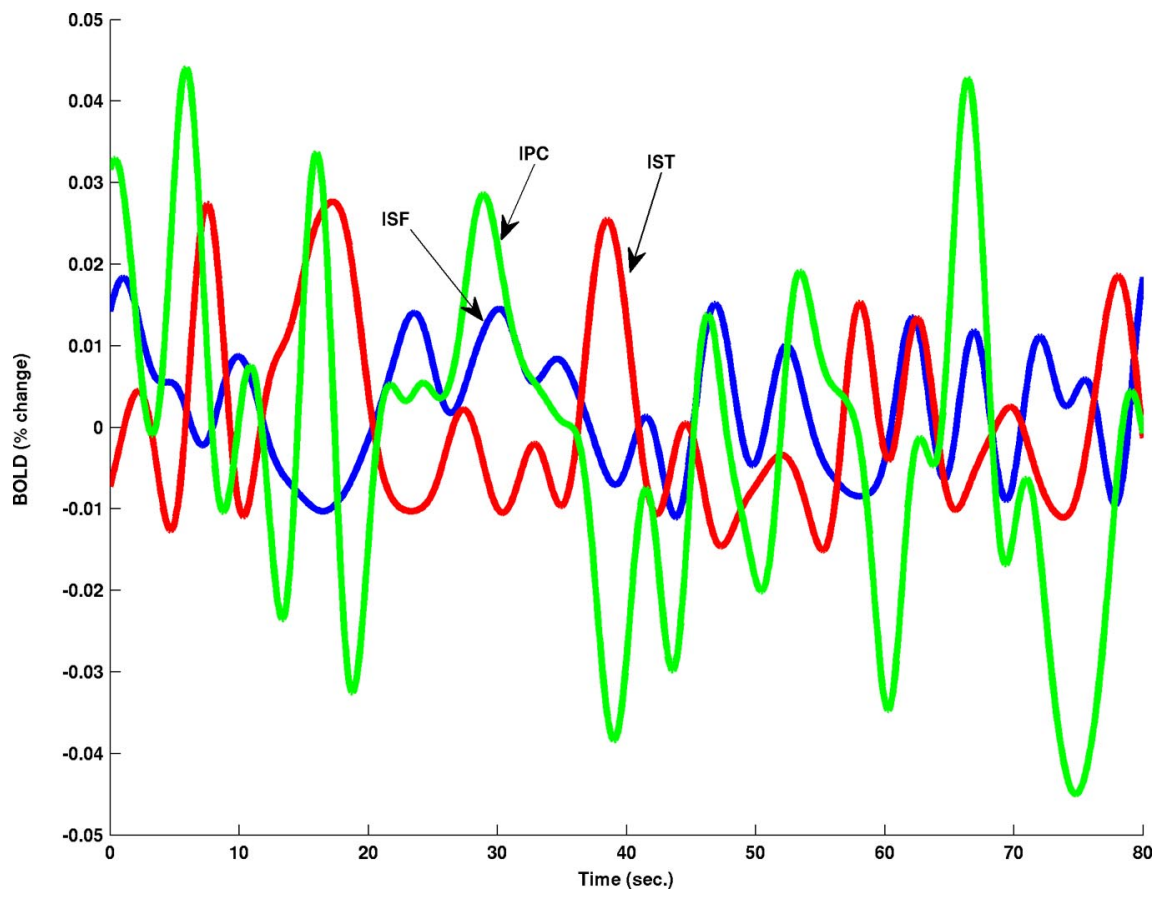

Figure 6. Simulated BOLDsignal for3 brain regions (IPC, green;ISF, blueand IST, red).IPCand ISFarepart of the Default Modenetwork, whereas IST is part of the Attentional Network. Both in theempirical and in thesimulated data, thefunctional connectivity between IPC and ISF is positive, while negative with IST. The temporal evolution of the signal shows this pattern of correlations. a
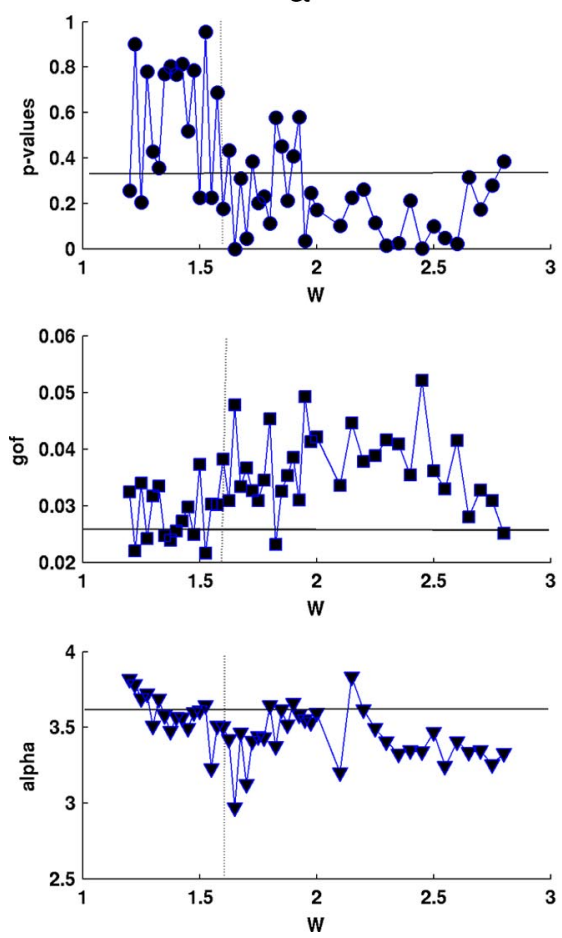

b
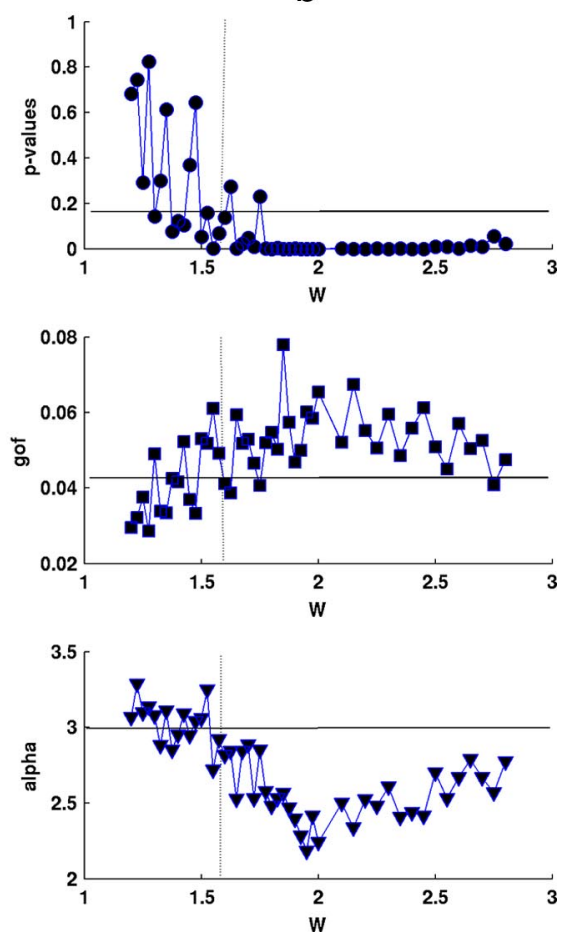

Figure 7. $p$-values of the Kolmogorov-Smirnov test, the gof, and the estimated maximum-likelihood estimate of the scaling exponent (alpha), as a function of the global coupling parameter W. a plots the results taking into account all pairs, i.e., including also the interhemispherical, whereas $\boldsymbol{b}$ plots the results for single hemispheres, i.e., excluding interhemispherical pairs. The horizontal line in each subpanel corresponds to the value obtained for the empirical data.

concept of resting state networks emerge at the level of slow fluctuations $\sim 0.1 \mathrm{~Hz}$.

At last, we show that at the edge of the bifurcation the tail of the distribution of the pair correlations between brain areas is maximally consistent with a power law distribution. To test the hypothesis that a given dataset is consistent with the assumption that they are drawn from a power-law distribution, we follow the standard goodness-of-fit test, which generates a $p$-value that quantifies the plausibility of the hypothesis. This test basically compares the "distance" between the distribution of the given dataset and the hypothesized power law distribution. Concretely we use here the method of Clauset et al. (2009) which first fit the given dataset to the power-law and calculate the Kolmogorov-Smirnov (KS) statistic for this fit. Next, we generate a large number of power-law distributed synthetic datasets with parameters equal to those of the distribution that best fit the given dataset. Each synthetic dataset is fit individually to its own power-law model and a KS statistic is obtained for each one relative to its own model. At last we count what fraction of the time the resulting statistic is larger than the value for the given dataset. This fraction establishes the $p$-value. If $p$ is large, then the difference between the given dataset and the assumed underlying power law model can be attributed to statistical fluctuations alone; if it is small $(<0.1)$, the power law model is not a plausible fit to the data. Figure 7 plots the $p$-values of the Kolmogorov-Smirnov test, the goodness of fit (gof), and the estimated maximumlikelihood estimate of the scaling exponent (alpha), as a function of the global coupling parameter $W$. As before Figure $7 a$ plots the results taking into account all pairs, i.e., including also the interhemispherical, whereas Figure $7 b$ plots the results for single hemispheres, i.e., excluding interhemispherical pairs. The horizontal line in each subpanel corresponds to the value obtained for the empirical data. A maximum of the $p$-value at the edge of the bifurcation is observed, indicating that at this point characteristic criticality behavior, like power law distributions, is observed. This is consistent with the recent analyses of Kitzbichler et al. (2009), who demonstrated criticality in empirical resting state data. Further more, the p-value, gof, and alpha estimated scaling exponent of the Kolmogorov-Smirnov test on the empirical functional connectivity data also gives a significant value and are very similar to the ones obtained in the simulations precisely at the brink of the bifurcation.

\section{Discussion}

In this paper, we investigated the neuronal and cortical mechanisms underlying the generation of resting state networks in the brain in 
absence of any stimulation and task. We formulated and studied a detailed and realistic spiking cortical network that is microscopically organized as standard attractor models (known from neural models of memory, attention, decision making, etc.) and mesoscopically organized through a neuroanatomical large-scale connectivity matrix obtained from human subjects via DTI/DSI tractography. More specifically, the model of a local brain area consists of integrateand-fire spiking neurons with excitatory (AMPA and NMDA) and inhibitory (GABA-A) synaptic receptor types. We demonstrated that the best fit of the empirical data as characterized by the AMRI BOLD signalbased functional connectivity is obtained when the brain network is critical. In other words, the brain network operates at the brink of a bifurcation that separates the stable equilibrium low activity state from the multistable state region where many attractors corresponding to high activity in different brain areas coexist. Under these conditions the slowly fluctuating $(<0.1 \mathrm{~Hz})$ resting state networks emerge as structured noise fluctuations around the stable low activity state induced by the presence of latent "ghost" multistable attractors at the edge of the bifurcation.

Conceptually the notion of a global attractor model of the brain network is not new (Grossberg, 1980; Hopfield, 1984; Carpenter and Grossberg, 2003; Haken, 2004). In these instances the equilibrium of a network is typically multistable, in other words different equilibrium states coexist and one state is finally realized. Ghosh et al. (2008) proposed originally that the RSN settles in one of these states and then explores in the presence of noise the neighborhood of this particular state. They have clearly demonstrated that the brain network must operate close to instability of the equilibrium to allow for such exploration. This phenomenon is called criticality. However, Ghosh et al. (2008) have not established the coexistence of multiple states, which will be eventually visited during the noise-driven exploration. Furthermore, if the brain network dynamics is indeed critical, then the multiple states may be stable, but they also may represent latent "ghost" attractors, which are regions of the state space just at the edge of the bifurcation. Answers to these questions will shed light not only on the mechanisms giving rise to the emergence of RSNs, but also have implications regarding our understanding of brain function.

In contrast to previous models (Honey et al., 2007; Ghosh et al., 2008; Deco et al., 2009) the spiking attractor model used here does not exhibit oscillations explicitly, but describes the firing rate of pools of neurons. Modulations of the neuronal subthreshold activity giving rise to rhythms as observed in local field potentials can be studied in principle, but have not been considered here, since we focused upon the emergent fix point attractors and their role in shaping the brain network's dynamic repertoire. This focus upon fix point attractors does not imply a static nature of the resting state network dynamics. On the contrary, the dynamics of the asynchronously firing neurons within a brain area is complex, but the fix point attractors prescribe its firing rate. The advantage of neglecting the oscillatory physiological signals lies in the fact that we can neglect the time delays via signal transmission between brain areas. This is justified in this particular case, because time delays do not alter the stationary attractor states of a network, however, they may alter the stability boundaries of the oscillatory network states (for review, see Jirsa, 2004; Jirsa and Ding, 2004; Jirsa, 2009). As a consequence, the resulting dynamic repertoire will be mostly determined by the properties of the neuroanatomical connectivity matrix. Through this approach we demonstrated that a large number of resting state networks, and therefore a large number of genuine attractors beyond the bifurcation threshold, is crucial for the size and richness of a network's dynamic repertoire.

It is intellectually tempting (and not too far-fetched, we believe) to interpret the multistable attractor landscape beyond the bifurca- tion point as functionally meaningful. When choosing the working point of the brain network at rest just below the bifurcation, then the multistable attractors express themselves as latent ghost attractors, i.e., the stable fixed points do not exist yet, but are either saddle points with at least one unstable direction, or regimes in the state space with slow flow. Criticality here means that the magnitude of the flow is close to zero at these points. As a consequence, these states can be easily stabilized when needed in a given task context or for a given function. This is in contrast to existing approaches in cognitive neurosciences, in which cognitive/behavioral states coexist and (task-specific) stimuli drive the system from one state to the other, or alternatively, state transitions are considered as mechanisms for goal-directed behavior (Kelso, 1995; Calvert et al., 2004; Haken, 2004; Kelso and Jirsa, 2004; Rolls and Deco, 2010). Our here proposed scenario is different: there exists a set of available brain states in a repertoire that can be rapidly activated. Even in absence of any task, the repertoire exists and expresses itself by shaping the dynamic flow in the neighborhood of the equilibrium point of the network. This subtle but crucial element establishes also the main difference of the here presented work and the model of Ghosh et al. (2008) with other RSN models. It is the fluctuations around the equilibrium point of the network that are structured, rather than the network state. In resting conditions, the system does not hop from a multistable state to another multistable state like in former models (Deco et al., 2009) but the fluctuations around the unique equilibrium (the low activity ground state) become structured induced by the attractor structure of the cortical model above the bifurcation point. The mechanism as proposed by Ghosh et al. (2008) is identical to the here proposed structured fluctuations, but it has never been established that the attractor landscape of Ghosh et al. (2008) is indeed multistable. This crucial multistability, however, we have demonstrated here explicitly. At the working point just below the critical threshold value, the brain network is not particularly prone to be in any other state than the equilibrium state, but with gentle external stimulation the system could be rapidly stabilized in one of the attractor states. The stabilization of a particular attractor will require well controlled, but minor reconfigurations of the network parameters rather than creating an attractor entirely from scratch. As we demonstrated here, the attractors are encoded in the connectivity matrix and are presumably associated with the computation of a specific brain function, which is evidenced by the fact that the resting state networks resemble intermittent activations of spatial network patterns that are consistently known to be activated under cognitive and behavioral task conditions (Fox et al., 2005). There have been several other studies, which demonstrated that in the absence of an overt task, spontaneous fluctuations in the BOLD signal correlate across functionally related brain regions (Lowe et al., 1998; Gusnard and Raichle, 2001; Greicius et al., 2003; Rogers et al., 2007). Some of these regions are also part of the Default Mode Network (Greicius et al., 2003), which identifies regions showing the greatest deactivation during externally imposed cognitive challenges. Interestingly multistable attractors (more accurately, bistable limit cycles) have been also postulated to be involved in the generation of the alpha rhythm (Freyer et al., 2011), which is a prominent mostly posterior rhythm of $8-12 \mathrm{~Hz}$ in the human. Alpha activity arises in absence of any stimulation or task context, in particular with eyes closed. Under conditions of noise, the corticothalamic model of Freyer et al. (2011) operates close to a subcritical Hopf bifurcation and captures various detailed temporal characteristics of alpha activity, including the bimodal distribution of the power spectrum and scale-invariant fluctuations. In its spirit, this modeling approach is closer to Deco et al. (2009) since it places the ongoing cortical oscillations beyond the threshold onto selfsustained oscillatory attractors (limit cycles). However, no spatio- 
temporal extension of this effort exists so far. Nevertheless, the detailed elaboration of the temporal characteristics of the alpha spectrum and the authors' conclusion that the brain operates close to instability is intriguing. In other words, the brain at rest appears to be critical.

We conclude that the multistable attractor landscape, as expressed by the latent ghost attractors during resting state conditions, defines a functionally meaningful dynamic repertoire of the brain network, that is inherently present in the neuroanatomical connectivity. This repertoire is functionally relevant, since it allows the system to rapidly compute a specific brain function through stabilization of one of its attractors. Consequently, the more entropy of attractors exists, the richer is the dynamical repertoire and therefore the brain network displays more capabilities of computation. We speculate therefore that the neuroanatomical connections developed a scale-free type of architecture to be able to store a large number of different and flexibly accessible brain functions. The numerous brain functions are evidenced indirectly under resting state conditions by the generation of a large diversity of networks reflecting different ways of structured fluctuations, i.e., by the resting state networks. These mechanisms will be studied in future works. An advantage of spiking models is precisely that we can incorporate effects like oscillations, neuromodulations, etc., in a simple way and without destroying the structure of attractors.

\section{References}

Assisi CG, Jirsa VK, Kelso JAS (2005) Synchrony and clustering in heterogeneous networks with global coupling and parameter dispersion. Phys Rev Lett 94:018106.

Attwell D, Buchan AM, Charpak S, Lauritzen M, Macvicar BA, Newman EA (2010) Glial and neuronal control of brain blood flow. Nature 468:232-243.

Biswal B, Yetkin FZ, Haughton VM, Hyde JS (1995) Functional connectivity in the motor cortex of resting human brain using echo-planar MRI. Magn Reson Med 34:537-541.

Brunel N, Wang XJ (2001) Effects of neuromodulation in a cortical network model of object working memory dominated by recurrent inhibition. J Comput Neurosci 11:63-85.

Bullmore E, Sporns O (2009) Complex brain networks: graph theoretical analysis of structural and functional systems. Nat Rev Neurosci 10:186-198.

Calvert GA, Spence S, Stein BE (2004) The handbook of multisenory processes. Cambridge, MA: MIT.

Carpenter GA, Grossberg S (2003) Adaptive resonance theory. In: The handbook of brain theory and neural networks (Arbib M, ed), pp 87-90. Cambridge, MA: MIT.

Clauset A, Shalizi C, Newman M (2009) Power-law distributions in empirical data. SIAM Rev 51:661-703.

Deco G, Jirsa V, McIntosh AR, Sporns O, Kötter R (2009) Key role of coupling, delay, and noise in resting brain fluctuations. Proc Natl Acad Sci U S A 106:10302-10307.

Deco G, Jirsa VK, McIntosh AR (2011) Emerging concepts for the dynamical organization of resting-state activity in the brain. Nat Rev Neurosci 12:43-56.

Fox MD, Raichle ME (2007) Spontaneous fluctuations in brain activity observed with functional magnetic resonance imaging. Nat Rev Neurosci $8: 700-711$.

Fox MD, Snyder AZ, Vincent JL, Corbetta M, Van Essen DC, Raichle ME (2005) The human brain is intrinsically organized into dynamic, anticorrelated functional networks. Proc Natl Acad Sci U S A 102:9673-9678.

Fransson P (2005) Spontaneous low-frequency BOLD signal fluctuations: an fMRI investigation of the resting-state default mode of brain function hypothesis. Hum Brain Mapp 26:15-29.

Freyer F, Roberts JA, Becker R, Robinson PA, Ritter P, Breakspear M (2011) Biophysical mechanisms of multistability in resting-state cortical rhythms. J Neurosci 31:6353-6361.

Friston KJ, Harrison L, Penny W (2003) Dynamic causal modelling. Neuroimage 19:1273-1302.

Ghosh A, Rho Y, McIntosh AR, Kötter R, Jirsa VK (2008) Noise during rest enables the exploration of the brain's dynamic repertoire. PLoS Comput Biol 4:e1000196.

Greicius MD, Krasnow B, Reiss AL, Menon V (2003) Functional connectivity in the resting brain: a network analysis of the default mode hypothesis. Proc Natl Acad Sci U S A 100:253-258.

Grossberg S (1980) Biological competition: decision rules, pattern formation and oscillations. Proc Natl Acad Sci U S A 77:2338-2342.

Gusnard D, Raichle ME (2001) Searching for a baseline: functional imaging and the resting human brain. Nat Rev Neurosci 2:685-694.

Hagmann P, Cammoun L, Gigandet X, Meuli R, Honey CJ, Wedeen VJ, Sporns O (2008) Mapping the structural core of human cerebral cortex. PLoS Biol 6:e159.

Haken H (2004) Synergetic computers and cognition: a top-down approach to neural nets. Berlin: Springer-Verlag.

Honey CJ, Kötter R, Breakspear M, Sporns O (2007) Network structure of cerebral cortex shapes functional connectivity on multiple time scales. Proc Natl Acad Sci U S A 104:10240-10245.

Honey CJ, Sporns O, Cammoun L, Gigandet X, Thiran JP, Meuli R, Hagmann P (2009) Predicting human resting-state functional connectivity from structural connectivity. Proc Natl Acad Sci U S A 106:2035-2040.

Hopfield JJ (1984) Neurons with graded response have collective computational properties like those of two-state neurons. Proc Natl Acad Sci U S A 81:3088-3092.

Izhikevich EM, Edelman GM (2008) Large-scale model of mammalian thalamocortical systems. Proc Natl Acad Sci U S A 105:3593-3598.

Jirsa VK (2004) Connectivity and dynamics of neural information processing. Neuroinformatics 2:183-204.

Jirsa VK (2009) Neural field dynamics with local and global connectivity and time delay. Philos Trans R Soc A 367:1131-1143.

Jirsa VK, Ding M (2004) Will a large complex system with time delays be stable? Phys Rev Lett 93:070602.

Jirsa VK, Stefanescu RA (2011) Neural population modes capture biologically realistic large scale network dynamics. Bull Math Biol 73:325-343.

Jirsa VK, Sporns O, Breakspear M, Deco G, McIntosh AR (2010) Towards the virtual brain: network modeling of the intact and the damaged brain. Arch Ital Biol 148:189-205.

Kelso JAS (1995) Dynamic patterns. The self-organization of brain and behavior. Cambridge, MA: MIT.

Kelso JAS, Jirsa VK, eds (2004) Coordination dynamics: issues and trends. Berlin: Springer.

Kitzbichler MG, Smith ML, Christensen SR, Bullmore E (2009) Broadband criticality of human brain networks synchronization. PLoS Comput Biol 5:e1000314.

Knock SA, McIntosh AR, Sporns O, Kötter R, Hagmann P, Jirsa VK (2009) The effects of physiologically plausible connectivity structure on local and global dynamics in large scale brain models. J Neurosci Methods 183:86-94.

Kötter R (2004) Online retrieval, processing, and visualization of primate connectivity data from the CoCoMac database. Neuroinformatics 2:127-144.

Logothetis NK, Pauls J, Augath M, Trinath T, Oeltermann A (2001) Neurophysiological investigation of the basis of the fMRI signal. Nature 412:150-157.

Lowe MJ, Mock BJ, Sorenson JA (1998) Functional connectivity in single and multislice echoplanar imaging using resting-state fluctuations. Neuroimage 7:119-132.

Raichle M, Mintun M (2006) Brain work and brain imaging, Annu Rev Neurosci 29:449-476.

Rogers BP, Morgan VL, Newton AT, Gore JC (2007) Assessing functional connectivity in the human brain by fMRI. Magn Reson Imaging 25:1347-1357.

Rolls E, Deco G (2010) The noisy brain. Oxford: Oxford UP.

Stefanescu RA, Jirsa VK (2008) A low dimensional description of globally coupled heterogeneous neural networks of excitatory and inhibitory neurons. PLoS Comput Biol 4:e1000219.

Stefanescu RA, Jirsa VK (2011) Reduced neural population dynamics with synaptic coupling. Phys Rev E Stat Nonlin Soft Matter Phys 83:026204.

Vincent JL, Patel GH, Fox MD, Snyder AZ, Baker JT, Van Essen DC, Zempel JM, Snyder LH, Corbetta M, Raichle ME (2007) Intrinsic functional architecture in the anaesthetized monkey brain. Nature 447:83-86.

Watts DJ, Strogatz SH (1998) Collective dynamics of 'small-world' networks. Nature 393:440-442. 